PROCEEDINGS OF THE

AMERICAN MATHEMATICAL SOCIETY

Volume 129, Number 3, Pages 823-831

S 0002-9939(00)05612-4

Article electronically published on September 20, 2000

\title{
A SIMPLE PROOF OF THE GROTHENDIECK THEOREM ON THE DIEUDONNÉ PROPERTY OF $C_{0}(T)$
}

\author{
T. V. PANCHAPAGESAN
}

(Communicated by Dale Alspach)

Dedicated to the memory of Professor Ivan Dobrakov

\begin{abstract}
Let $T$ be a locally compact Hausdorff space and let $C_{0}(T)$ be the Banach space of all complex valued continuous functions vanishing at infinity in $T$, provided with the supremum norm. Let $X$ be a locally convex Hausdorff space (briefly, an lcHs) which is quasicomplete. A simple proof of the Grothendieck theorem on the Dieudonné property of $C_{0}(T)$ is given. The present proof is much simpler than that given in an earlier work of the author (Characterizations of weakly compact operators on $C_{0}(T)$, Trans. Amer. Math. Soc. 350 (1998), 4849-4867).
\end{abstract}

\section{INTRODUCTION}

Let $T$ be a locally compact Hausdorff space and $C_{0}(T)$ the Banach space of all complex valued continuous functions $f$ vanishing at infinity in $T$, endowed with the supremum norm $\|f\|_{T}=\sup _{t \in T}|f(t)|$. Let $M(T)$ be the dual of $C_{0}(T)$, consisting of all bounded complex Radon measures on $T$, with their domain restricted to the $\sigma$-algebra of all Borel sets in $T$. Let $X$ be a locally convex Hausdorff space (briefly, an $\mathrm{lcHs}$ ), which is quasicomplete.

In [14 we observed that, contrary to Remark 2 of 7], the Grothendieck techniques in [7] are not powerful enough to prove the locally compact analogue of Theorem 6 of [7] if the locally compact space is not further $\sigma$-compact and hence the Dieudonné property of $C_{0}(T)$ for $T$ arbitrary remained unsettled until the publication of our paper [13]. Employing new techniques, we not only obtained in [13] the locally compact analogue of Theorem 6 of [7] (which establishes the Dieudonné property of $C_{0}(T)$ ), but also provided several new characterizations for a continuous linear map $u: C_{0}(T) \rightarrow X$ to be weakly compact. The proof given in [13] makes use of Lemma 1 and Theorem 2 of [7], Theorem 1 of [13] (which provides a technique, similar to that of Bartle-Dunford-Schwartz in [1], for the lcHs case) and Theorems 1 and 2 of [12, which characterize relatively weakly compact sets in $M(T)$ in terms of the Baire, $\sigma$-Borel and Borel restrictions of the members of the set in question. The proof of Theorem 1 of [12] is quite involved and deep.

Received by the editors January 22, 1999 and, in revised form, May 24, 1999.

1991 Mathematics Subject Classification. Primary 47B38, 46G10; Secondary 28B05.

This research was supported by the project C-845-97-05-B of the C.D.C.H.T. of the Universidad de los Andes, Mérida, Venezuela. 
In this note we dispense with the use of Theorems 1 and 2 of [12] and give a simple proof of the Grothendieck theorem on the Dieudonné property of $C_{0}(T)$. The present proof is based on Lemma 1 and Theorem 2 of [7], the first part of Theorem 1 of [13] and the theorem on regular Borel extension of $X$-valued Baire measures on $T$. We obtain in Theorem 1 below, excepting four, all the remaining 31 characterizations given in [13] for a continuous linear map $u: C_{0}(T) \rightarrow X$ to be weakly compact and the Dieudonné property of $C_{0}(T)$ is an immediate consequence of the equivalence of the assertions (1) and (8) or of (1) and (34) in the said theorem. Of course, the present techniques devoid of the use of Theorem 1 of [12] are not powerful enough to obtain all the 35 characterizations given in [13] (see Remark 3). But, on the other hand, the new characterizations (19), (20) and (21) of Theorem 1 below are also deducible by the techniques of 13 if one invokes the equivalence of (i) and (ii) of Proposition 1 of [13].

\section{Preliminaries}

In this section we fix notation and terminology. For the convenience of the reader we also give some definitions and results from [5, 11, 13].

In the sequel $T, C_{0}(T),\|\cdot\|_{T}, M(T)$ and $X$ will be as stated in Introduction.

Let $\mathcal{K}\left(\right.$ resp. $\left.\mathcal{K}_{o}\right)$ be the family of all compacts (resp. compact $G_{\delta} \mathrm{s}$ ) in $T$. The $\sigma$-ring $\mathcal{B}_{o}(T)$ (resp. $\mathcal{B}_{c}(T)$ ) of all Baire (resp. $\sigma$-Borel) sets in $T$ is the $\sigma$-ring generated by $\mathcal{K}_{o}$ (resp. $\mathcal{K}$ ). The $\sigma$-algebra $\mathcal{B}(T)$ of all Borel sets in $T$ is the $\sigma$ algebra generated by the class of all open sets in $T$. Note that a subset $E$ of $T$ is $\sigma$-Borel if and only if it is a $\sigma$-bounded Borel set in $T$, thereby justifying our terminology.

Recall that $M(T)$ is the Banach space of all bounded complex Radon measures on $T$ with their domain restricted to $\mathcal{B}(T)$ so that each $\mu \in M(T)$ is a regular (bounded) complex Borel measure on $T$ and has norm given by $\|\mu\|=$ $\operatorname{var}(\mu, \mathcal{B}(T))(T)$. For $\mu \in M(T),|\mu|(E)=\operatorname{var}(\mu, \mathcal{B}(T))(E)$, for $E \in \mathcal{B}(T)$.

We recall the following result from [13 Lemma 1].

Proposition 1. For $\mu \in M(T)$,

$$
|\mu|_{\mathcal{B}_{o}(T)}(\cdot)=\operatorname{var}\left(\left.\mu\right|_{\mathcal{B}_{o}(T)}, \mathcal{B}_{o}(T)\right)(\cdot) \text { and }|\mu|_{\mathcal{B}_{c}(T)}(\cdot)=\operatorname{var}\left(\left.\mu\right|_{\mathcal{B}_{c}(T)}, \mathcal{B}_{c}(T)\right)(\cdot) \text {. }
$$

A vector measure is an additive set function defined on a ring of sets with values in an lcHs. In the sequel $X$ denotes an lcHs with topology $\tau$. $\Gamma$ is the set of all $\tau$-continuous seminorms on $X$. The dual of $X$ is denoted by $X^{*}$.

The strong topology $\beta\left(X^{*}, X\right)$ of $X^{*}$ is the locally convex topology induced by the seminorms $\left\{p_{B}: B\right.$ bounded in $\left.X\right\}$, where

$$
p_{B}\left(x^{*}\right)=\sup _{x \in B}\left|x^{*}(x)\right| .
$$

$X^{* *}$ denotes the dual of $\left(X^{*}, \beta\left(X^{*}, X\right)\right)$ and is endowed with the locally convex topology $\tau_{e}$ of uniform convergence on equicontinuous subsets of $X^{*}$. Note that $\left(X^{*}, \beta\left(X^{*}, X\right)\right)$ and $\left(X^{* *}, \tau_{e}\right)$ are lcHs.

It is well known that the canonical injection $J: X \rightarrow X^{* *}$ given by $\left\langle J x, x^{*}\right\rangle=$ $\left\langle x, x^{*}\right\rangle$ for all $x \in X$ and $x^{*} \in X^{*}$ is linear. On identifying $X$ with $J X \subset X^{* *}$, one has $\left.\tau_{e}\right|_{J X}=\left.\tau_{e}\right|_{X}=\tau$.

Let $\mathcal{E}=\left\{A \subset X^{*}: A\right.$ is equicontinuous $\}$. Then the family of seminorms $\Gamma_{\mathcal{E}}=\left\{p_{A}: A \in \mathcal{E}\right\}$ induces the topology $\tau$ of $X$ and the topology $\tau_{e}$ of $X^{* *}$, where $p_{A}(x)=\sup _{x^{*} \in A}\left|x^{*}(x)\right|$ for $x \in X$ and $p_{A}\left(x^{* *}\right)=\sup _{x^{*} \in A}\left|x^{* *}\left(x^{*}\right)\right|$ for $x^{* *} \in X^{* *}$. 
Definition 1. A linear map $u: C_{0}(T) \rightarrow X$ is called a weakly compact operator on $C_{0}(T)$ if $\left\{u f:\|f\|_{T} \leq 1\right\}$ is relatively weakly compact in $X$.

The following result is the same as Lemma 2 of [13], where the hypothesis of quasicompleteness of $X$ is redundant.

Proposition 2. Let $X$ be an lcHs and let $u: C_{0}(T) \rightarrow X$ be a continuous linear map. Then $u^{*} A$ is bounded in $M(T)$ for each $A \in \mathcal{E}$.

For each $\tau$-continuous seminorm $p$ on $X$, let $p(x)=\|x\|_{p}, x \in X$.

Let $\mathcal{S}$ be a $\sigma$-ring of subsets of a nonempty set $\Omega$. An $X$-valued vector measure $m$ on $\mathcal{S}$ is said to be bounded if $\{m(E): E \in \mathcal{S}\}$ is bounded in $X$.

For the theory of integration of bounded $\mathcal{S}$-measurable scalar functions with respect to a bounded quasicomplete lcHs-valued vector measure defined on the $\sigma$ ring $\mathcal{S}$, the reader may refer to [11] or [13. We need the following results from Lemma 6 of [11] and Proposition 7 of [13].

Proposition 3. Let $X$ be a quasicomplete lcHs and let $\mathcal{S}$ be a $\sigma$-ring of subsets of R. Then:

(i) If $f$ is a bounded $\mathcal{S}$-measurable scalar function and $m$ is an $X$-valued bounded vector measure on $\mathcal{S}$, then $f$ is $m$-integrable and

$$
x^{*}\left(\int_{\Omega} f d m\right)=\int_{\Omega} f d\left(x^{*} \circ m\right)
$$

for each $x^{*} \in X^{*}$.

(ii) (Lebesgue bounded convergence theorem) If $m$ is an $X$-valued $\sigma$-additive vector measure on $\mathcal{S}$ and $\left(f_{n}\right)$ is a bounded sequence of $\mathcal{S}$-measurable scalar functions with $\lim _{n} f_{n}(w)=f(w)$ for each $w \in \Omega$, then $f$ is $m$-integrable and

$$
\int_{E} f d m=\lim _{n} \int_{E} f_{n} d m
$$

for each $E \in \mathcal{S}$.

The following result is due to the first part of Theorem 1 of [13] which is analogous to Theorem VI.2.1 of $\left[2\right.$ for lcHs-valued continuous linear maps on $C_{0}(T)$. It plays a vital role in Section 3 .

Proposition 4. Let $X$ be an lcHs and let $u: C_{0}(T) \rightarrow X$ be a continuous linear map. Then there exists a unique $X^{* *}$-valued vector measure $m$ on $\mathcal{B}(T)$ satisfying the following properties:

(i) $x^{*} \circ m \in M(T)$ for each $x^{*} \in X^{*}$ and consequently, $m: \mathcal{B}(T) \rightarrow X^{* *}$ is $\sigma$-additive in the $\sigma\left(X^{* *}, X^{*}\right)$-topology.

(ii) The mapping $x^{*} \rightarrow x^{*} \circ m$ of $X^{*}$ into $M(T)$ is weak ${ }^{*}$-weak ${ }^{*}$ continuous. Moreover, $u^{*} x^{*}=x^{*} \circ m, x^{*} \in X^{*}$.

(iii) $x^{*} u f=\int_{T} f d\left(x^{*} \circ m\right)$ for each $f \in C_{0}(T)$ and $x^{*} \in X^{*}$.

(iv) The range of $m$ is $\tau_{e}$-bounded in $X^{* *}$.

(v) $m(E)=u^{* *}\left(\chi_{E}\right)$ for $E \in \mathcal{B}(T)$.

Definition 2. Let $u: C_{0}(T) \rightarrow X$ be a continuous linear map. Then the vector measure $m$ as given in Proposition 4 is called the representing measure of $u$.

Definition 3. A $\sigma$-additive vector measure $m: \mathcal{B}_{o}(T) \rightarrow X$ (resp. $\mathcal{B}(T) \rightarrow X$, $\mathcal{B}_{c}(T) \rightarrow X$ ) is called an $X$-valued Baire (resp. Borel, $\sigma$-Borel) measure on $T$. 
Definition 4. Let $\mathcal{S}$ be a $\sigma$-ring of sets in $T$ with $\mathcal{S} \supset \mathcal{K}$ or $\mathcal{K}_{o}$. Let $m: \mathcal{S} \rightarrow X$ be a vector measure. Then $m$ is said to be $\mathcal{S}$-regular (resp. $\mathcal{S}$-outer regular, $\mathcal{S}$-inner regular) in $E \in \mathcal{S}$ if, given a seminorm $p \in \Gamma$ and an $\epsilon>0$, there exist a compact set $K \in \mathcal{S}$ and an open set $U \in \mathcal{S}$ with $K \subset E \subset U$ (resp. an open set $U \in \mathcal{S}$ with $E \subset U$, a compact set $K \in \mathcal{S}$ with $K \subset E)$ such that $\|m(B)\|_{p}<\epsilon$ for all $B \in \mathcal{S}$ with $B \subset U \backslash K$ (resp. $\|m(B)\|_{p}<\epsilon$ for all $B \in \mathcal{S}$ with $B \subset U \backslash E,\|m(B)\|_{p}<\epsilon$ for all $B \in \mathcal{S}$ with $B \subset E \backslash K)$. Even though $T$ does not belong to $\mathcal{S}$, one can define $\mathcal{S}$-inner regularity of $m$ in $T$ as follows. Given $p \in \Gamma$ and $\epsilon>0$, there exists a compact $K \in \mathcal{S}$ such that $\|m(B)\|_{p}<\epsilon$ for all $B \in \mathcal{S}$ with $B \subset T \backslash K$. The vector measure $m$ is said to be $\mathcal{S}$-regular (resp. $\mathcal{S}$-outer regular, $\mathcal{S}$-inner regular) if it is so in each $E \in \mathcal{S}$. When $\mathcal{S}=\mathcal{B}(T)\left(\right.$ resp. $\mathcal{B}_{o}(T), \mathcal{B}_{c}(T)$ ), we use the terminology Borel (resp. Baire, $\sigma$-Borel) regularity or outer regularity or inner regularity.

Remark 1 . In the above definition one can replace $\Gamma$ by any other family of $\tau$ continuous seminorms on $X$ which induces the topology $\tau$.

The following proposition is well known and plays a key role in the next section. It was first proved in [4, 9] for Banach spaces and extended to group-valued measures in [15. See [16] for semigroup valued measures. For a simple and direct proof, see [5].

Proposition 5. Let $m$ be an $X$-valued Baire measure on $T$ and let $X$ be a quasicomplete lcHs. Then $m$ is Baire regular. Moreover, there exists a unique $X$-valued Borel (resp. $\sigma$-Borel) regular $\sigma$-additive extension $\hat{m}$ (resp. $\hat{m}_{c}$ ) of $m$ on $\mathcal{B}(T)$ (resp. $\mathcal{B}_{c}(T)$ ). Moreover, $\left.\hat{m}\right|_{\mathcal{B}_{c}(T)}=\hat{m}_{c}$.

\section{Characterizations of Weakly compact operators on $C_{0}(T)$}

In the following definition we weaken the second part of Definition 4 of [7].

Definition 5. Let $X$ be an lcHs. The first Baire class of $X^{* *}$ is the subspace of $X^{* *}$ formed by the $\sigma\left(X^{* *}, X^{*}\right)$-limits of $\sigma\left(X, X^{*}\right)$-Cauchy sequences of elements in $X$. Let $H$ be the first Baire class of $X^{* *}$. Then we say that $X$ has the Dieudonné property if for each quasicomplete lcHs $Y$, each continuous linear map $u: X \rightarrow Y$ with $u^{* *}(H) \subset Y$ satisfies $u^{* *}\left(X^{* *}\right) \subset Y$.

Lemma 1. Let $m: \mathcal{B}(T) \rightarrow X^{* *}$ be a vector measure and let $m_{c}=\left.m\right|_{\mathcal{B}_{c}(T)}$ and $m_{o}=\left.m\right|_{\mathcal{B}_{o}(T)}$. If $m$ (resp. $m_{c}, m_{o}$ ) is Borel (resp. $\sigma$-Borel, Baire) inner regular $\left(\right.$ in $\left.\tau_{e}\right)$ in $\mathcal{B}(T)$ (resp. $\mathcal{B}_{c}(T), \mathcal{B}_{o}(T)$ ), then $m$ (resp. $\left.m_{c}, m_{o}\right)$ is $\sigma$-additive in $\tau_{e}$.

Proof. Let $A$ be an equicontinuous subset of $X^{*}$ and let $\epsilon>0$. Let $\mathcal{S}=\mathcal{B}(T)$ and $\gamma=m\left(\right.$ resp. $\mathcal{S}=\mathcal{B}_{c}(T)$ and $\gamma=m_{c} ; \mathcal{S}=\mathcal{B}_{o}(T)$ and $\left.\gamma=m_{o}\right)$. Let $\left(E_{n}\right)_{1}^{\infty}$ be a nonincreasing sequence in $\mathcal{S}$ with $\bigcap_{1}^{\infty} E_{n}=\emptyset$. By hypothesis, for each $n$, there exists a compact $K_{n} \in \mathcal{S}$ with $K_{n} \subset E_{n}$ such that $\sup _{x^{*} \in A}\left|\left(x^{*} \circ \gamma\right)(B)\right|<\frac{\epsilon}{2^{n}}$ for all $B \in \mathcal{S}$ with $B \subset E_{n} \backslash K_{n}$. Then it follows that $\sup _{x^{*} \in A} \operatorname{var}\left(x^{*} \circ \gamma, \mathcal{S}\right)\left(E_{n} \backslash K_{n}\right)<\frac{4 \epsilon}{2^{n}}$ for all $n$. Now adapting the proof of $(\mathrm{d}) \Rightarrow(\mathrm{e})$ of Lemma 13 on pp.158-159 of [2], one can show that there exists $n_{o}$ such that $\sup _{x^{*} \in A} \operatorname{var}\left(x^{*} \circ \gamma, \mathcal{S}\right)\left(E_{n}\right)<4 \epsilon$ for all $n \geq n_{o}$. Consequently, $\left\|\gamma\left(E_{n}\right)\right\|_{p_{A}}<4 \epsilon$ for $n \geq n_{o}$ and hence $\gamma$ is $\sigma$-additive in $\tau_{e}$.

Theorem 1. $C_{0}(T)$ has the Dieudonné property. More precisely, let $u: C_{0}(T) \rightarrow$ $X$ be a continuous linear map, where $X$ is a quasicomplete lcHs. Let $m$ be the representing measure of $u$, and let $m_{c}=\left.m\right|_{\mathcal{B}_{c}(T)}$ and $m_{o}=\left.m\right|_{\mathcal{B}_{o}(T)}$. Then the 
following assertions are equivalent:

(1) $u$ is weakly compact.

(2) The range of $m$ is contained in $X$.

(3) The range of $m_{c}$ is contained in $X$.

(4) The range of $m_{o}$ is contained in $X$.

(5) $m(U) \in X$ for all open sets $U$ in $T$.

(6) $m(F) \in X$ for all closed sets $F$ in $T$.

(7) $m(U) \in X$ for all $\sigma$-Borel open sets $U$ in $T$.

(8) $m(U) \in X$ for all open Baire sets $U$ in $T$.

(9) $m(U) \in X$ for all open sets $U$ in $T$ which are $\sigma$-compact.

(10) $m(F) \in X$ for all closed sets $F$ in $T$ which are $G_{\delta}$.

(11) $m(U) \in X$ for all open sets $U$ in $T$ which are $F_{\sigma}$.

(12) For a nondecreasing sequence $\left(f_{n}\right)_{1}^{\infty} \subset C_{0}(T)$, with $0 \leq f_{n} \leq 1$, $\left(u f_{n}\right)$ converges weakly in $X$.

(13) $m$ is $\sigma$-additive in the topology $\tau_{e}$ of $X^{* *}$.

(14) $m_{c}$ is $\sigma$-additive in the topology $\tau_{e}$ of $X^{* *}$.

(15) $m_{o}$ is $\sigma$-additive in the topology $\tau_{e}$ of $X^{* *}$.

(16) $m$ is strongly additive in the topology $\tau_{e}$ of $X^{* *}$.

(17) $m_{c}$ is strongly additive in the topology $\tau_{e}$ of $X^{* *}$.

(18) $m_{o}$ is strongly additive in the topology $\tau_{e}$ of $X^{* *}$.

(19) $m$ is exhaustive in the family of all open sets in $T$ with respect to the topology $\tau_{e}$ of $X^{* *}$ in the sense that for each disjoint sequence $\left(U_{n}\right)_{1}^{\infty}$ of open sets in $T, \lim _{n} m\left(U_{n}\right)=0$.

(20) $m_{c}$ is exhaustive in the family of all $\sigma$-Borel open sets in $T$ with respect to the topology $\tau_{e}$ of $X^{* *}$.

(21) $m_{o}$ is exhaustive in the family of all open Baire sets in $T$ with respect to the topology $\tau_{e}$ of $X^{* *}$.

(22) $m$ is Borel regular in $\tau_{e}$ of $X^{* *}$.

(23) $m$ is Borel inner regular in $\tau_{e}$ of $X^{* *}$.

(24) $m$ is Borel outer regular (in $\tau_{e}$ ) in each compact set $K$ in $T$ and Borel inner regular (in $\tau_{e}$ ) in the set $T$.

(25) $m_{c}$ is $\sigma$-Borel regular in $\tau_{e}$ of $X^{* *}$.

(26) $m_{c}$ is $\sigma$-Borel inner regular in $\tau_{e}$ of $X^{* *}$.

(27) $m_{c}$ is $\sigma$-Borel outer regular (in $\tau_{e}$ ) in each compact set $K$ in $T$ and $\sigma$-Borel inner regular (in $\tau_{e}$ ) in the set $T$.

(28) $m_{o}$ is Baire regular in $\tau_{e}$ of $X^{* *}$.

(29) $m_{o}$ is Baire inner regular in $\tau_{e}$ of $X^{* *}$.

(30) All bounded Borel measurable scalar functions $f$ on $T$ are $m$-integrable and $\int_{T} f d m \in X$.

(31) All bounded $\mathcal{B}_{c}(T)$-measurable scalar functions $f$ on $T$ are $m$-integrable and $\int_{T} f d m_{c} \in X$.

(32) All bounded $\mathcal{B}_{o}(T)$-measurable scalar functions $f$ on $T$ are $m$-integrable and $\int_{T} f d m_{o} \in X$.

(33) All bounded scalar functions $f$ belonging to the first Baire class in $T$ are $m_{o^{-}}$ integrable and $\int_{T} f d m_{O} \in X$.

(34) $u^{* *} f \in X$ for all bounded scalar functions $f$ belonging to the first Baire class in $T$.

Proof. In the following we shall prove only those implications which are not obvious. 
$(1) \Rightarrow(2)$ by Corollary 9.3.2 of Edwards [6], by Proposition 4(v) and by the fact that $\chi_{E} \in C_{0}^{* *}(T)$ for all $E \in \mathcal{B}(T)$.

$(8) \Rightarrow(1)$ In fact, on the contrary, by Corollary 9.3.2 of Edwards [6] there would exist an equicontinuous set $A$ in $X^{*}$ such that $u^{*}(A)$ is not relatively weakly compact in $M(T)$. As $u^{*}(A)$ is bounded in $M(T)$ by Proposition 2, by (3) of Theorem 4.22.1 of [6] there would exist a disjoint sequence $\left(U_{n}\right)_{1}^{\infty}$ of open sets in $T$ and an $\epsilon>0$ such that $\sup _{x^{*} \in A}\left|\left(x^{*} \circ m\right)\left(U_{n}\right)\right|>\epsilon$ for all $n$. Consequently, there would exist $x_{n}^{*} \in A$ such that $\left|\left(x_{n}^{*} \circ m\right)\left(U_{n}\right)\right|>\epsilon$ for all $n$.

Since $x_{n}^{*} \circ m \in M(T)$ by Proposition $4(\mathrm{i}), x_{n}^{*} \circ m$ is Borel regular in $U_{n}$ and therefore there exists a compact $K_{n} \subset U_{n}$ such that $\left|\left(x_{n}^{*} \circ m\right)\left(K_{n}\right)\right|>\epsilon$ for all $n$. Let $D\left(K_{n}\right)=\left\{U: U\right.$ open, $\left.K_{n} \subset U \subset U_{n}\right\}$ and let $U \geq V$ for $U, V \in D\left(K_{n}\right)$ if $U \subset V$. Then, as $x_{n}^{*} \circ m$ is outer Borel-regular in $K_{n}$, we have

$$
\lim _{U \rightarrow K_{n}, U \in D\left(K_{n}\right)}\left(x_{n}^{*} \circ m\right)(U)=\left(x_{n}^{*} \circ m\right)\left(K_{n}\right) .
$$

Thus there exists an open set $W_{n} \in D\left(K_{n}\right)$ such that $\left|\left(x_{n}^{*} \circ m\right)(V)\right|>\epsilon$ for all $V \in D\left(K_{n}\right)$ with $V \subset W_{n}$. As $K_{n} \subset W_{n}$, by Theorem 50.D of Halmos [8] there exists an open Baire set $V_{n}$ such that $K_{n} \subset V_{n} \subset W_{n}$ so that $\left|\left(x_{n}^{*} \circ m\right)\left(V_{n}\right)\right|>\epsilon$. Thus we have found open Baire sets $\left(V_{n}\right)_{1}^{\infty}$ in $T$ such that $K_{n} \subset V_{n} \subset U_{n}$ for all $n$ and such that $\left|\left(x_{n}^{*} \circ m\right)\left(V_{n}\right)\right|>\epsilon$ for all $n$. Let $V=\bigcup_{1}^{\infty} V_{n}$. Then $V$ is an open Baire set and by hypothesis (8) we have $m(V) \in X$ and $m\left(V_{n}\right) \in X$ for all $n$. Now by (i) of Proposition 4 and by the Orlicz-Pettis theorem for lcHs (see [10]) we conclude that $m(V)=\sum_{1}^{\infty} m\left(V_{n}\right)$, the series converging unconditionally in the topology $\tau$ of $X$. As $\tau=\left.\tau_{e}\right|_{X}$, it follows particularly that $p_{A}\left(m\left(V_{n}\right)\right) \rightarrow 0$ as $n \rightarrow \infty$. But, on the other hand, $p_{A}\left(m\left(V_{n}\right)\right)=\sup _{x^{*} \in A}\left|\left(x^{*} \circ m\right)\left(V_{n}\right)\right| \geq\left|\left(x_{n}^{*} \circ m\right)\left(V_{n}\right)\right|>\epsilon$ for all $n$. This contradiction proves that $u$ is weakly compact and hence (1) holds.

$(10) \Rightarrow(11)$ Let $U$ be open and $F_{\sigma}$ in $T$. Then $T \backslash U$ is closed and $G_{\delta}$. Then by (10) we have $m(U)=m(T)-m(T \backslash U) \in X$. Hence (11) holds.

$(9) \Rightarrow(8)$ by $\S 14$ of Dinculeanu 3 .

$(2) \Rightarrow(12)$ Let $\left(f_{n}\right)$ be as in $(12)$. Then $\lim _{n} f_{n}(t)=f(t)$ exists in $[0,1]$ for each $t \in T$ and $f$ is Borel measurable. Then by (2), by Proposition 4(i) and by the Orlicz-Pettis theorem for lcHs, $m$ has range in $X$ and is $\sigma$-additive (in $t$ ) in $\mathcal{B}(T)$. Then by Proposition 3(ii) we have $\lim _{n} \int_{T} f_{n} d m=\int_{T} f d m \in X$. Consequently, by Propositions 3(i) and 4(iii)

$$
\lim _{n} x^{*} u f_{n}=\lim _{n} \int_{T} f_{n} d\left(x^{*} \circ m\right)=x^{*}\left(\lim _{n} \int_{T} f_{n} d m\right)=x^{*}\left(\int_{T} f d m\right)
$$

for all $x^{*} \in X^{*}$. Thus (12) holds.

$(12) \Rightarrow(8)$ Let $U$ be an open Baire set in $T$. Then by $\S 14$ of Dinculeanu 3 there exists a sequence $\left(K_{n}\right) \subset \mathcal{K}_{o}$ such that $K_{n} \nearrow U$. By Urysohn's lemma we can choose a nondecreasing sequence $g_{n}$ of nonnegative continuous functions with compact supports such that $g_{n} \nearrow \chi_{U}$. Then by hypothesis there exists a vector $x_{o} \in X$ such that $\lim _{n} x^{*} u g_{n}=x^{*} x_{o}$ for all $x^{*} \in X^{*}$. Therefore, by Proposition 4(iii) and by the Lebesgue dominated convergence theorem we have $x^{*} x_{o}=\lim _{n} \int_{T} g_{n} d\left(x^{*} \circ m\right)=x^{*} m(U)$ for all $x^{*} \in X^{*}$. As $m(U) \in X^{* *}$, it follows that $m(U)=x_{o} \in X$. Hence (8) holds.

$(2) \Rightarrow(13)$ by Proposition 4(i), by the Orlicz-Pettis theorem for lcHs and by the fact that $\left.\tau_{e}\right|_{X}=\tau$.

$(21) \Rightarrow(1)$ In fact, on the contrary, following the argument in the proof of $(8) \Rightarrow(1)$, we have an $\epsilon>0$, an equicontinuous subset $A$ of $X^{*}$, a disjoint sequence $\left(V_{n}\right)_{1}^{\infty}$ of 
open Baire sets in $T$ and a sequence $\left(x_{n}^{*}\right)_{1}^{\infty}$ in $A$ such that $\left|\left(x_{n}^{*} \circ m\right)\left(V_{n}\right)\right|>\epsilon$ for all $n$. This contradicts the hypothesis that $\lim _{n}\left\|m\left(V_{n}\right)\right\|_{p_{A}}=0$. Hence (1) holds.

$(2) \Rightarrow(22)$ (resp. (25), (28)) By (2), Proposition 4(i) and the Orlicz-Pettis theorem for lcHs, $m$ is $\sigma$-additive on $\mathcal{B}(T)$ in the topology $\tau$ of $X$. Then $m_{o}$ is $\sigma$-additive on $\mathcal{B}_{o}(T)$ and has range in $X$. Therefore, by the first part of Proposition $5, m_{o}$ is regular and hence (28) holds. Moreover, by the second part of Proposition 5, there exists a unique $X$-valued $\sigma$-additive (in $\tau$ ) regular Borel extension $\hat{m}$ of $m_{o}$ on $\mathcal{B}(T)$ and $\hat{m}_{c}=\left.\hat{m}\right|_{\mathcal{B}_{c}(T)}$ is $\sigma$-Borel regular. Then by Proposition 4 (iii) and by the fact that each $f \in C_{0}(T)$ is bounded and Baire measurable (see Theorem 51.B of [8]), we have

$$
x^{*} u f=\int_{T} f d\left(x^{*} \circ m\right)=\int_{T} f d\left(x^{*} \circ m_{o}\right)=\int_{T} f d\left(x^{*} \circ \hat{m}\right)
$$

for each $x^{*} \in X^{*}$ and $f \in C_{0}(T)$. Since $x^{*} \circ m$ and $x^{*} \circ \hat{m}$ are in $M(T)$ and represent the continuous linear functional $x^{*} u$ on $C_{0}(T)$, we conclude that $x^{*} \circ m=x^{*} \circ \hat{m}$ for all $x^{*} \in X^{*}$. Since $m$ has range in $X^{* *}$ and $\hat{m}$ in $X$, it follows that $m=\hat{m}$ and hence $(22)$ (resp. (25)) holds.

$(22) \Rightarrow(27)$ Given $K \in \mathcal{K}, A \in \mathcal{E}$ and $\epsilon>0$, by hypothesis there exists an open set $U$ with $U \supset K$ such that $\|m(B)\|_{p_{A}}<\epsilon$ for all $B \in \mathcal{B}(T)$ with $B \subset U \backslash K$. By Theorem 50.D of Halmos [8], we can choose an open Baire set $V$ in $T$ such that $K \subset V \subset U$ so that $\|m(B)\|_{p_{A}}<\epsilon$ for all $B \in \mathcal{B}(T)$ with $B \subset V \backslash K$ and hence, particularly, $\left\|m_{c}(B)\right\|_{p_{A}}<\epsilon$ for all $B \in \mathcal{B}_{c}(T)$ with $B \subset V \backslash K$. Thus $m_{c}$ is $\sigma$-Borel outer regular in $K$. Clearly, $m_{c}$ is $\sigma$-Borel inner regular in $T$ as by hypothesis (22) $m$ is Borel inner regular in $T$. Thus (27) holds.

By Lemma 1 , we have $(23) \Rightarrow(13),(26) \Rightarrow(14)$ and $(29) \Rightarrow(15)$.

$(24) \Rightarrow(1)$ Let $K \in \mathcal{K}$ and let $A$ be an equicontinuous set in $X^{*}$. Given $\epsilon>0$, by hypothesis there exists an open set $U$ in $T$ such that $K \subset U$ and $\|m(B)\|_{p_{A}}<\epsilon$ for all $B \in \mathcal{B}(T)$ with $B \subset U \backslash K$. Since $u^{*} x^{*}=x^{*} \circ m$, we have

$$
\sup _{x^{*} \in A}\left|\left(x^{*} \circ m\right)\right|(U \backslash K) \leq 4 \epsilon .
$$

Thus condition (4)(a) of Theorem 4.22.1 of Edwards [6] is satisfied by $u^{*}(A)$. Again by hypothesis, there exists $C \in \mathcal{K}$ such that $\|m(B)\|_{p_{A}}<\epsilon$ for all $B \in \mathcal{B}(T)$ with $B \subset T \backslash C$ so that $\sup _{x^{*} \in A}\left|\left(x^{*} \circ m\right)\right|(T \backslash C) \leq 4 \epsilon$. Hence condition (4)(b) of the said theorem is also satisfied by $u^{*} A$. Since $u^{*} A$ is bounded in $M(T)$ by Proposition 2 , we conclude by Theorem 4.22 .1 of 6 ] that $u^{*} A$ is relatively weakly compact in $M(T)$ and hence, by Corollary 9.3 .2 of Edwards [6], $u$ is weakly compact.

$(27) \Rightarrow(1)$ Let $K \in \mathcal{K}$. Proceeding as in the proof of $(24) \Rightarrow(1)$, there exists a $\sigma$-Borel open set $U$ containing $K$ such that $\sup _{x^{*} \in A}\left|\left(x^{*} \circ m_{c}\right)(B)\right|<\epsilon$, for all $B \in \mathcal{B}_{c}(T)$ with $B \subset U \backslash K$. Then, we have

$$
\sup _{x^{*} \in A} \operatorname{var}\left(x^{*} \circ m_{c}, \mathcal{B}_{c}(T)\right)(U \backslash K)<4 \epsilon .
$$

Therefore, by Proposition 1, $\sup _{x^{*} \in A}\left|\left(x^{*} \circ m\right)\right|(U \backslash K)<4 \epsilon$ where $\left|\left(x^{*} \circ m\right)\right|=$ $\operatorname{var}\left(x^{*} \circ m, \mathcal{B}(T)\right)$. Thus, condition 4(a) of Theorem 4.22.1 of Edwards [6] is satisfied by $u^{*} A$. Again by hypothesis, there exists a compact $C$ such that $\left\|m_{c}(B)\right\| p_{p_{A}}<\epsilon$ for all $B \in \mathcal{B}_{c}(T)$ with $B \subset T \backslash C$. Thus $\sup _{x^{*} \in A}\left|\left(x^{*} \circ m\right)(K)\right|<\epsilon$, for each compact $K \subset T \backslash C$. Then by the Borel regularity of $x^{*} \circ m$ it follows that

$$
\sup _{x^{*} \in A}\left|\left(x^{*} \circ m\right)\right|(T \backslash C) \leq 4 \epsilon .
$$


Thus $u^{*} A$ also satisfies condition 4(b) of Theorem 4.22.1 of Edwards [6]. Since $u^{*} A$ is bounded in $M(T)$ by Proposition 2, by Theorem 4.22.1 of [6] $u^{*} A$ is relatively weakly compact in $M(T)$. Hence, by Corollary 9.3.2 of [6], (1) holds.

Obviously, (2) implies (30) since $m$ is $\tau_{e}(=\tau)$-bounded by Proposition 4(iv). $(32) \Rightarrow(8)$ as the hypothesis implies that $m(U) \in X$ for every open Baire set $U$ in $T$. As shown in the proof of $(12) \Rightarrow(8), \chi_{U}$ belongs to the first Baire class for each open Baire set $U$ in $T$ and hence $(33) \Rightarrow(8)$ (resp. $(34) \Rightarrow(8)$ as $u^{* *}\left(\chi_{U}\right)=m(U)$ ). By Corollary 9.3.2 of Edwards [6], (1) implies that the range of $u^{* *}$ is contained in $X$ and hence $(1) \Rightarrow(34)$.

In the light of Corollary 9.3.2 of Edwards [6], the equivalence of (1) and (34) implies that $C_{0}(T)$ has the Dieudonné property.

This completes the proof of the theorem.

Remark 2. Corollary 9.3.2 of Edwards [ [6] is essentially due to Lemma 1 of [7], and Theorem 4.22.1 of [6] is the same as Theorem 2 of [7].

Remark 3. The techniques used in the above proof are not strong enough to prove the equivalences of the statements (i), (xxi), (xxv), (xxix) and (xxx) given in 13 . But, on the other hand, the new characterizations (19), (20) and (21) of Theorem 1 above are also deducible by the techniques of [13] if one appeals to the equivalence of (i) and (ii) of Proposition 1 of [13].

\section{ADDED IN PROOF}

An alternative proof, also based on the Borel extension theorem, is provided in our recent paper "A Borel extension approach to weakly compact operators in $C_{0}(T)$ " (to appear in Czechoslovak Math. J.) to obtain all the 35 characterizations given in [13].

\section{REFERENCES}

[1] R. G. Bartle, N. Dunford and J. T. Schwartz, Weak compactness and vector measures, Canad. J. Math. 7, (1955), 289-305. MR 16:1123c

[2] J. Diestel and J. J. Uhl, Vector Measures, Survey No.15, Amer. Math. Soc., Providence, R.I., (1977). MR 56:12216

[3] N. Dinculeanu, Vector Measures, Pergamon Press, New York, (1967). MR 34:6011b

[4] N. Dinculeanu and I. Kluvánek, On vector measures, Proc. London Math. Soc. 17, (1967), 505-512. MR 35:5571

[5] I. Dobrakov and T. V. Panchapagesan, A simple proof of the theorem on Borel extension and weak compactness of operators, submitted.

[6] R. E. Edwards, Functional Analysis, Theory and Applications, Holt, Rinehart and Winston, New York, (1965). MR 36:4308 corrected reprint MR 95k:46001

[7] A. Grothendieck, Sur les applications linéares faiblement compactes d'espaces du type $C(K)$, Canad. J. Math. 5, (1953), 129-173. MR 15:438b

[8] P. R. Halmos, Measure Theory, Van Nostrand, New York, (1950). MR 11:504d

[9] I. Kluvánek, Characterization of Fourier-Stieltjes transforms of vector and operator valued measures, Czech. Math. J. 17, (1967), 261-277. MR 37:6430

[10] C. W. McArthur, On a theorem of Orlicz and Pettis, Pacific J. Math. 22, (1967), 297-302. MR 35:4702

[11] T. V. Panchapagesan, Applications of a theorem of Grothendieck to vector measures, J. Math. Anal. Appl. 214, (1997), 89-101. MR 99h:28022

[12] T. V. Panchapagesan, Baire and $\sigma$-Borel characterizations of weakly compact sets in $M(T)$, Trans. Amer. Math. Soc. 350 (1998), 4839-4847. MR 99h:28008

[13] T. V. Panchapagesan, Characterizations of weakly compact operators on $C_{0}(T)$, Trans. Amer. Math. Soc. 350, (1999), 4849-4867. MR 99b:47046 
[14] T. V. Panchapagesan, On the limitations of the Grothendieck techniques, to appear in Rev. Real Acad. Cienc. Exact. Fis. Natur. Madrid.

[15] M. Sion, Outer measures with values in a topological group, Proc. London Math. Soc., 19, (1969), 89-106. MR 39:398

[16] H. Weber, Fortsetzung von Massen mit Werten in uniformen Halbgruppen, Arch. Math., XXVII, (1976), 412-423. MR 54:13028

Departamento de Matemáticas, Facultad de Ciencias, Universidad de los Andes, MÉrida, Venezuela

E-mail address: panchapa@ciens.ula.ve 